Received: 18 April 2020 | Accepted: 4 May 2020 | First published online: 23 May 2020

DOI: 10.1002/ijgo.13191

\title{
Effectiveness of a COVID-19 screening questionnaire for pregnant women at admission to an obstetric unit in Milan
}

\author{
Beatrice Tassis $^{1}$ | Giovanna Lunghi ${ }^{2}$ | Maria P. Frattaruolo ${ }^{1}$ | \\ Marta Ruggiero $^{1,3}$ | Edgardo Somigliana ${ }^{1,3, *}$ | Enrico Ferrazzi ${ }^{1,3}$ \\ ${ }^{1}$ Department of Women, Newborns, and Children, Fondazione IRCCS Ca' Granda Ospedale Maggiore Policlinico, Mangiagalli Center, Milan, Italy \\ ${ }^{2}$ Virology Unit, Fondazione IRCCS Ca' Granda Ospedale Maggiore Policlinico, Mangiagalli Center, Milan, Italy \\ ${ }^{3}$ Department of Clinical Sciences and Community Health, University of Milan, Milan, Italy \\ ${ }^{*}$ Correspondence \\ Edgardo Somigliana, Fondazione IRCCS Ca' Granda Ospedale Maggiore Policlinico, Milan, Italy. \\ Email: dadosomigliana@yahoo.it
}

KEYWORDS: Coronavirus; COVID-19; Obstetrics unit; SARS-Cov-2; Screening

Sutton et al. ${ }^{1}$ recently reported on universal testing with nasopharyngeal swabs to detect severe acute respiratory syndrome coronavirus 2 (SARS-Cov-2) infection in 215 women admitted for delivery at the Presbyterian Allen Hospital in New York, USA. They identified 33 (15.3\%) infected women, of whom only four had fever or symptoms suggesting coronavirus disease 2019 (COVID-19). These findings suggest that only universal testing can reliably recognize infected cases. However, this approach is only feasible in major hospitals in high-resource countries with efficient lab facilities in-house. Alternative approaches deserve consideration.

The Fondazione IRCCS Ca' Granda Ospedale Maggiore Policlinico is a designated COVID-19 maternity hub, located in Milan, northern Italy-an area severely hit by SARS-Cov- 2 . $^{2}$ Since the early phases of the outbreak and in line with local recommendations, we opted for systematic screening for SARS-Cov-2 infection using a specific questionnaire at obstetrics admission (Fig. 1). ${ }^{3}$ Suspected cases based on this questionnaire underwent nasopharyngeal swab and were managed as a suspected COVID-19 case until the result of the test became available. Patients were admitted in a dedicated COVID-19 area and managed by properly trained and protected personnel. Patients with unremarkable questionnaire results were managed according to current standards.

To validate this approach, we performed nasopharyngeal swabs from April 1-9, 2020 for all pregnant women requiring hospital admission. A total of 139 consecutive women were included. Overall, 6
(4.3\%) women were considered suspected cases by the screening questionnaire, while the remaining 133 (95.7\%) were not. Nasopharyngeal swab results were positive in $3(2.2 \%)$ women, two among the suspected cases and one among the women with unremarkable screening responses $(P=0.005)$. The prevalence of COVID-19 among the women with unremarkable questionnaire responses was $0.8 \%(95 \%$ $\mathrm{Cl}, 0.1-4.1)$.

Our findings suggest that, at the time of this observational study, thanks to early lockdown, Milan was facing a different phase and severity of the outbreak compared with New York. ${ }^{1}$ Even if the study periods overlap, the rate of positive swabs in the two areas were radically different (2.2\% vs $15.3 \%)$. The performance of our screening approach might be less efficient in areas with a similar situation as New York, where the absolute rate of undetected COVID-19 cases would be markedly higher.

In conclusion, a policy of systematic screening with a specific questionnaire is a manageable, inexpensive, and effective tool in obstetric care, at least in areas where the incidence of SARS-Cov-2 infection is not devastating. However, it is not infallible. If swabs can be processed within a matter of hours, a universal swab policy is preferred.

\section{AUTHOR CONTRIBUTIONS}

EF and BT designed the study. GL, MPF and MR collected the data. ES wrote the first draft. All the authors revised the manuscript. 


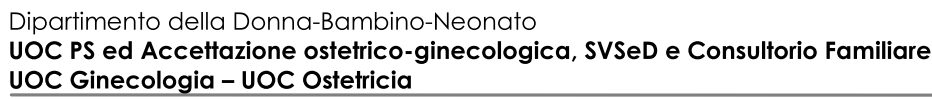

\section{CHECK-LIST TO DETECT SARS-CoV-2 SYMPTOMATOLOGY AND POSSIBLE CONTACTS WITH INFECTION RISK}

NAME and SURNAME

1) Do you have a SARS-CoV-2 positive swab

2) Symptoms: in the last 14 days have you had one of the following symptoms:

○ Temperature $\geq 37.5^{\circ} \mathrm{C}$

○ Cough

- Sore throat or cold

- Headache

Diarrhea and/or vomit

Dyspnea (breathing difficulty) / Tachypnea (increase in respiratory rate)

- Asthenia (weakness) e/o arthromyalgia (muscular pain associated with a joint)

- Anosmia (alteration in the sense of smell)

- Ageusia (alteration in the sense of taste)

- None of the above

- No information available

3) In the last 14 days:

- Have you had a high risk job (laboratory technician, health worker, etc)

- Have you been in close contact with COVID-19 positive people

- Have you lived with a person affected by a flu-like syndrome/pneumonia or with a person in a high risk job (laboratory technician, health worker, etc)

- Have you taken part in meetings that have become outbreaks of flu-like syndrome/pneumonia ( $\geq 2$ affected people)

- None of the above

- No information available

On the basis of anamnestic data (if one or more symptoms/risk factors are present)

SENDING TO AN AREA FOR COVID-19 SUSPECT PATIENTS

Date

Physician's signature

Patient's signature

Da: 1) "ISUOG Interim Guidance on 2019 novel coronavirus infection during pregnancy and puerperium: information for healthcare professionals. Ultrasound Obstet Gynecol. 2020 Mar 11"- Modificato; 2) Infezione da SARS-CoV-2: indicazioni ad interim per gravida-partoriente, puerpera-neonato e allattamento. Regione Lombardia Marzo 2020

ISTITUTO DI RICOVERO E CURA A CARATTERE SCIENTIFICO DI NATURA PUBBLICA

Via Francesco Sforza, 28 - 20122 Milano

Tel. 025503.1 - www.policlinico.mi.it - CF e P.I. 04724150968

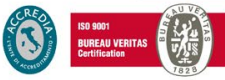

Polo di ricerca, cura e formazione universitaria UNIVERSITÀ DEGLI STUD

FIGURE 1 Checklist used to assess pregnant women with suspected COVID-19 at hospital admission. The English version is presented but translations in nine different languages were available. The checklist was adapted from Poon et al. ${ }^{3}$ 
CONFLICTS OF INTEREST

The authors have no conflicts of interest.

\section{REFERENCES}

1. Sutton D, Fuchs K, D'Alton M, Goffman D. Universal screening for SARS-CoV-2 in women admitted for delivery. N Engl J Med. 2020; [Epub ahead of print].
2. Grasselli G, Pesenti A, Cecconi M. Critical care utilization for the COVID-19 outbreak in Lombardy, Italy. JAMA. 2020;323(16):1545.

3. Poon LC, Yang H, Lee JC, et al. ISUOG Interim Guidance on 2019 novel coronavirus infection during pregnancy and puerperium: information for healthcare professionals. Ultrasound Obstet Gynecol. 2020; [Epub ahead of print].

\title{
Critically ill pregnant patient with COVID-19 and neonatal death within two hours of birth
}

\author{
Jianwei $\mathrm{Li}^{1 \ddagger} \mid$ Yichun Wang ${ }^{2 \ddagger} \mid$ Yingchun Zeng ${ }^{3 \ddagger} \mid$ Ting Song ${ }^{4} \mid$ Xingfei Pan $^{5}$ | \\ Mingwang $\mathrm{Jia}^{2}$ | Fang $\mathrm{He}^{3}$ | Liusheng $\mathrm{Hou}^{1}$ | Bingfei $\mathrm{Li}^{6^{*}}$ | Shuming $\mathrm{He}^{7^{*}}$ | Dunjin Chen ${ }^{3^{*}}$ \\ ${ }^{1}$ Department of Critical Care, Zhongshan People's Hospital, Zhongshan, Guangdong Province, China \\ ${ }^{2}$ Department of Critical Care, The Third Affiliated Hospital of Guangzhou Medical University, Guangzhou, China \\ ${ }^{3}$ Research Institute of Gynecology and Obstetrics, The Third Affiliated Hospital of Guangzhou Medical University, Guangzhou, China \\ ${ }^{4}$ Department of Radiology, The Third Affiliated Hospital of Guangzhou Medical University, Guangzhou, China \\ ${ }^{5}$ Department of Infectious Disease, The Third Affiliated Hospital of Guangzhou Medical University, Guangzhou, China \\ ${ }^{6}$ Department of Anesthesiology, Zhongshan People's Hospital, Zhongshan, Guangdong Province, China \\ ${ }^{7}$ Xiaolan People's Hospital of Zhongshan, Zhongshan, Guangdong Province, China \\ ${ }^{*}$ Correspondence \\ Bingfei Li, Shuming He and Dunjin Chen, Research Institute of Gynecology and Obstetrics, The Third Affiliated Hospital of Guangzhou Medical University, \\ Guangdong Provincial Key Laboratory of Obstetrics Major Diseases, Guangzhou, China. \\ Email: gzdrchen@gzhmu.edu.cn \\ ${ }^{\ddagger}$ These authors contributed equally.
}

KEYWORDS: China; Coronavirus; COVID-19; Pregnancy; Neonatal death; SARS-Cov-2

While most pregnant women with coronavirus disease 2019 (COVID-19) appear to experience a milder clinical course, ${ }^{1,2}$ the present report describes a critical case of COVID-19 in a pregnant woman. We discuss the identification, diagnosis, disease progression, and treatment outcome in a 31-year-old pregnant woman admitted to Xiaolan People's Hospital of Zhongshan at 35+2 weeks of pregnancy with no known comorbidity or history of chronic illness. Onset of symptoms in the patient began with a sore throat and dry cough for 4 days, followed by fever and dyspnea for half a day. The timeline of the patient's disease history and illness progression is shown in Figure 1. The patient experienced rapid aggravation of the disease.
Emergency cesarean delivery was performed at the bedside, but the neonate died within two hours of birth (Fig. 2).

Although the patient had no history of chronic disease, the severity of COVID-19 increased rapidly-from dyspnea to acute respiratory distress syndrome and septic shock within 12 hours. The patient's condition worsened, with persistent decreases in white blood cell and lymphocyte counts. Inflammation indicators of C-reactive protein, procalcitonin, and interleukin 6 all increased significantly, whereas peripheral oxygen saturation level decreased progressively. Given these circumstances, white blood cell and lymphocyte counts of COVID-19 patients should be monitored 